Session 1520

\title{
Use of Graphical Programming Tools for Electrical Engineering and Technology Courses
}

\author{
Salahuddin Qazi and Naseem Ishaq \\ School of Information Systems and Engineering Technology \\ State University of New York Institute of Technology \\ Utica, New York 13504.
}

\begin{abstract}
The design and implementation of algorithms based on graphical language or block diagram programming tools using PCs make a design and its implementation easier, faster and is a natural way of expressing certain algorithms. The increasing use of these software tools in industry makes it important to prepare the electrical engineering and engineering technology students for the changing industrial environment. The purpose of this paper is to discuss the use of three graphical programming language tools : STK of AGI Inc., SignalPro of EDX Inc., and System View of ELANIX Inc., as teaching aids in electrical engineering technology courses at the State University of New York Institute of Technology, Utica, NY. All the three software tools are PC based and are available free of cost to the qualifying colleges and universities for classroom usage. Other graphical programming tools used by the authors in the electrical engineering technology courses are also reviewed.
\end{abstract}

\section{Introduction}

Graphical or block diagram programming is a method of writing custom programs based on placing and connecting a variety of graphical functions or block diagrams. It differs from textual or traditional programming where a specialized syntax based text is needed to structure a program. Graphical programming tools are increasingly used to simulate a design, develop an algorithm or model a process in engineering and technology disciplines due to ease of implementation and because they allow a natural, intuitive interaction with the system or process under simulation. The traditional approach to develop and implement an algorithm to target a real-time DSP is to write the source code in either $\mathrm{C}$ language or assembly language. Such an approach although doable is laborious, time consuming, requires great deal of programming expertise, and detracts a designer from the process of developing the actual design to be implemented. In graphical programming, it is easier for a designer to design, simulate and implement the algorithms expressed as block diagrams. One of the most important advantage of graphical programming is the possibility of building large programs graphically, especially by students, engineers and scientists with a little formal knowledge of the 
programming. Graphical programming also has an important role in simulating and modeling of virtual labs which has become essential with the popularity of web-based instruction for distance learning. An experimental facility based on virtual labs allows students to investigate and examine variations in problems that are difficult or sometimes impossible to duplicate in the physical labs. It is also possible to offer students these virtual labs via the world wide web or CD-ROM anytime and anywhere in the world ${ }^{1,2}$.

\section{Review of Graphical Programming Tools}

Due to many advantages of graphical programming, the industry has developed a range of software tools for different areas of electrical engineering. and technology. As a result, the electrical engineering technology department at the SUNY Institute of Technology, has introduced a number of software packages to prepare the students for the changing industrial environment. Some of these packages are reviewed below:

LABVIEW $^{3}$ of National Instruments was first implemented in 1986 by providing a graphical tool for measurements tasks in the area of laboratory automation. It contains an extensive library of functions, libraries for data acquisition, data presentation and data storage. Its four basic operations include virtual instruments, front panels, block diagrams and icon and connectors.

SIMULINK of MathWorks ${ }^{4}$ Inc., provides a graphical block diagram simulation environment for modeling, simulating, and analyzing dynamic systems. It can be used to build graphical block diagrams, evaluate system performance, and is integrated with MATLAB and Stateflow for modeling event-driven behavior. It is used interactively for DSP design, communications system design and control system design as well as for other simulation applications.

SystemView of ELANIX ${ }^{5}$ Inc., is a comprehensive dynamic systems analysis environment for the design and simulation of engineering or scientific systems. It provides sophisticated analysis engine for digital signal processing, RF/Analog, filter design, control system, communications systems and other general mathematical systems. It combines an intuitive block diagram interface and extensive libraries for easy system design.

EDX SignalPro is based on powerful wireless network planning tools developed by the EDX ${ }^{6}$ division of Comarco Wireless Technologies, Inc. It is a general purpose software package for wireless communication systems from $30 \mathrm{MHz}$ to $60 \mathrm{GHz}$ and includes multi-site coverage and interference analysis for a comprehensive set of propagation models. It also has full mapping capabilities, and full access to terrain, groundcover (clutter), building, demographic, traffic, and other databases. Optional modules are available to provide specialized requirement of LMDS, MMDS, WLL, and PCS/cellular system design, as well as 3-D outdoor / indoor ray-tracing studies.

Proceeding of the 2002 American Society for Engineering Education Annual Conference \& Exposition Copyright (0) 2002, American Society for Engineering Education 
RIDE (Real-time Integrated Design Environment) of Hypersignal ${ }^{7}$ software is based on graphical DSP environment that provides component-based design for real time DSP algorithm development. RIDE allows the user to create a DSP algorithm from a block diagram approach and facilitates the design, implementation, and analysis of real time DSP algorithms and systems. It includes an automatic ANSI C source code generator for generating $\mathrm{C}$ source code from graphical design block diagram.

OPNET $^{8}$ (Optimized Network Engineering Tools) is a PC based graphical simulation tool that can create and manipulate protocols of communication networks including optical and wireless networks. It is organized in a hierarchical structure of modes consisting of network models, nodes models, process modes and parameter models. OPNET's radio module provides capability of modeling radio links and mobile communications nodes including ground, airborne and satellite systems.

STK ${ }^{9}$ (Satellite Tool Kit) of Analytical Graphics, Inc., is a leading of-the-shelf commercial satellite systems analysis software for the aerospace industry. It is the core product of the STK software suite and provides the analytical engine to calculate data and display multiple 2-D maps to visualize various time-dependent information for satellites and other space-related objects such as launch vehicles, missiles, and aircraft. Its basic applications include calculating and visualizing a vehicle's position and attitude, determining acquisition times, and analyzing the vehicle's field of view. For specific analytical tasks, several STK add-on modules are available from AGI to support problems such as detailed communication link analysis, radar analysis, orbit determination, coverage analysis, maneuver planning, and real-time integration.

RSOFT ${ }^{10}$ (Research Software) Inc., has developed a CAD based software for the photonics and fiber optics industry. It consist of three products, namely BeamPROP for simulation of integrated and fiber-based photonic devices, FullWAVE for finite difference time domain analysis of complex photonic devices, and LinkSIM for simulation of optical links for telecommunications and data communications.

\section{Teaching with Graphical Programming Tools}

The authors have used most of these software packages reviewed above in their research and also in their classroom teaching. The professional copies of some of these software packages are expensive and require annual maintenance fee for licensing. This paper will describe the following three PC based graphical programming tools used by the authors in their electrical engineering technology courses. These packages were donated to the college for classroom usage.

1. STK of AGI Inc.

2. SignalPro of EDX Inc.

3. System View of Elanix Inc.

Proceeding of the 2002 American Society for Engineering Education Annual Conference \& Exposition Copyright (C) 2002, American Society for Engineering Education 
1. STK of AGI was used for the satellite communication course which starts with the basic understanding of Kepler's laws governing the satellite motion and builds on the principles of satellite communication technology. The STK software is used to create satellite orbits. Seven orbit selections including sun synchronous, geostationary, Molniya, repeating ground trace, critically inclined, and circular are available. With the proper parameters, study of low earth orbit (LEO), medium earth orbit(MEO), highly elliptical orbit (HEO), was also performed. The following figure shows an example of Molniya and circular orbits. The orbits were also animated.

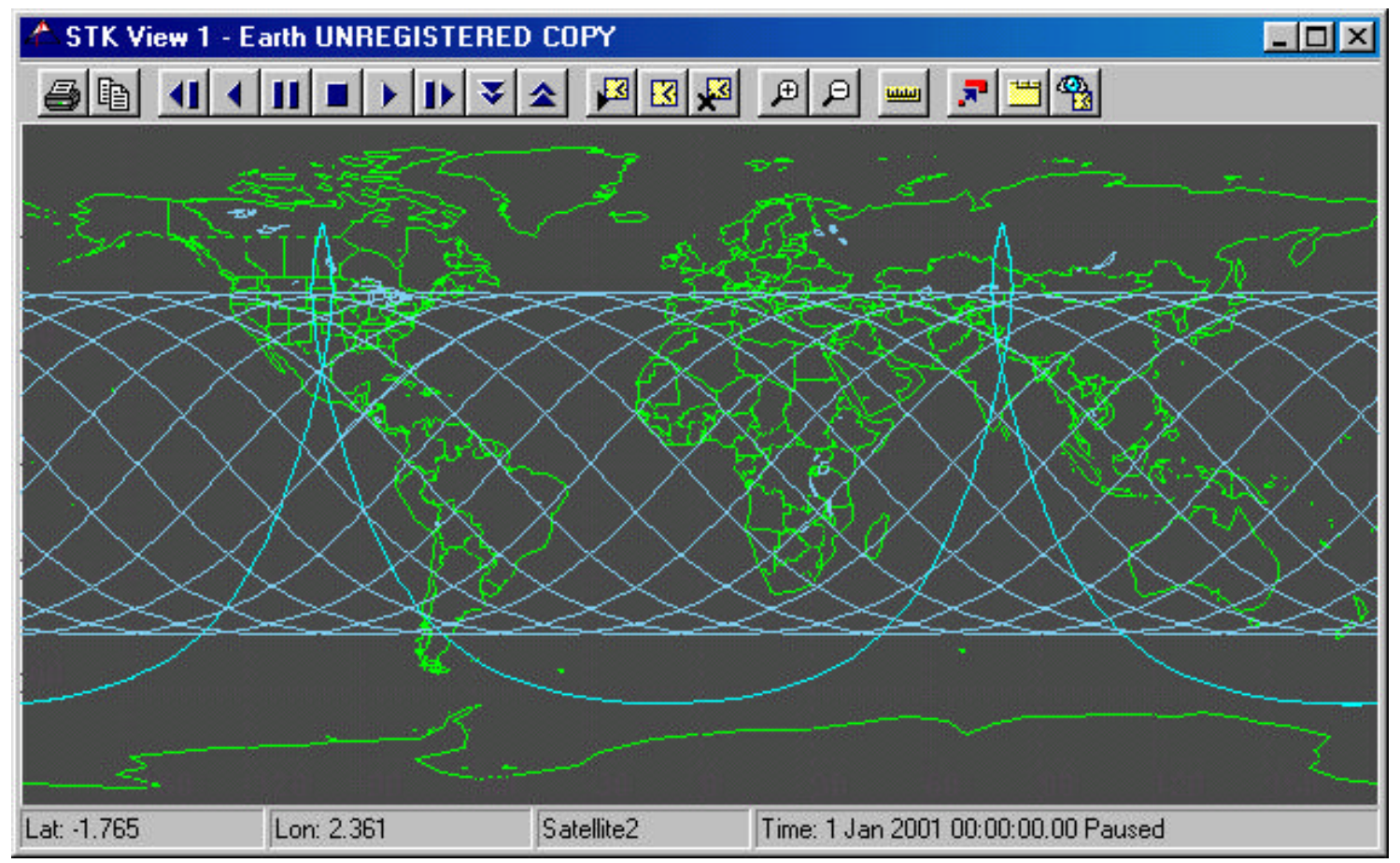

The STK website (http://www.stk.com) contains a lot of useful material, software downloads, animations, models, satellite database and astrogator examples. AGI Inc., also offers Educational Alliance Program, under which the eligible colleges, universities, military training institutions, high schools and non-profit educational groups can obtain STK products with a license for classroom use The faculty can incorporate hands-on experience with STK software into their curriculum, helping students' marketability as they enter the work force.

2. The SignalPro of EDX Inc., was used in the course on Wireless Communication Systems to perform area study which allows drawing, maps of signal levels and interference for cellular communication services to predict performance. It can also be used for other communication services like two-way mobile, paging, multipoint, and PCS. It also allows the user to select from different study types, numerous propagation models, specifying thousands of transmitter sites and displaying the results in a variety of ways. The example of the area study for three sector link is given below. 


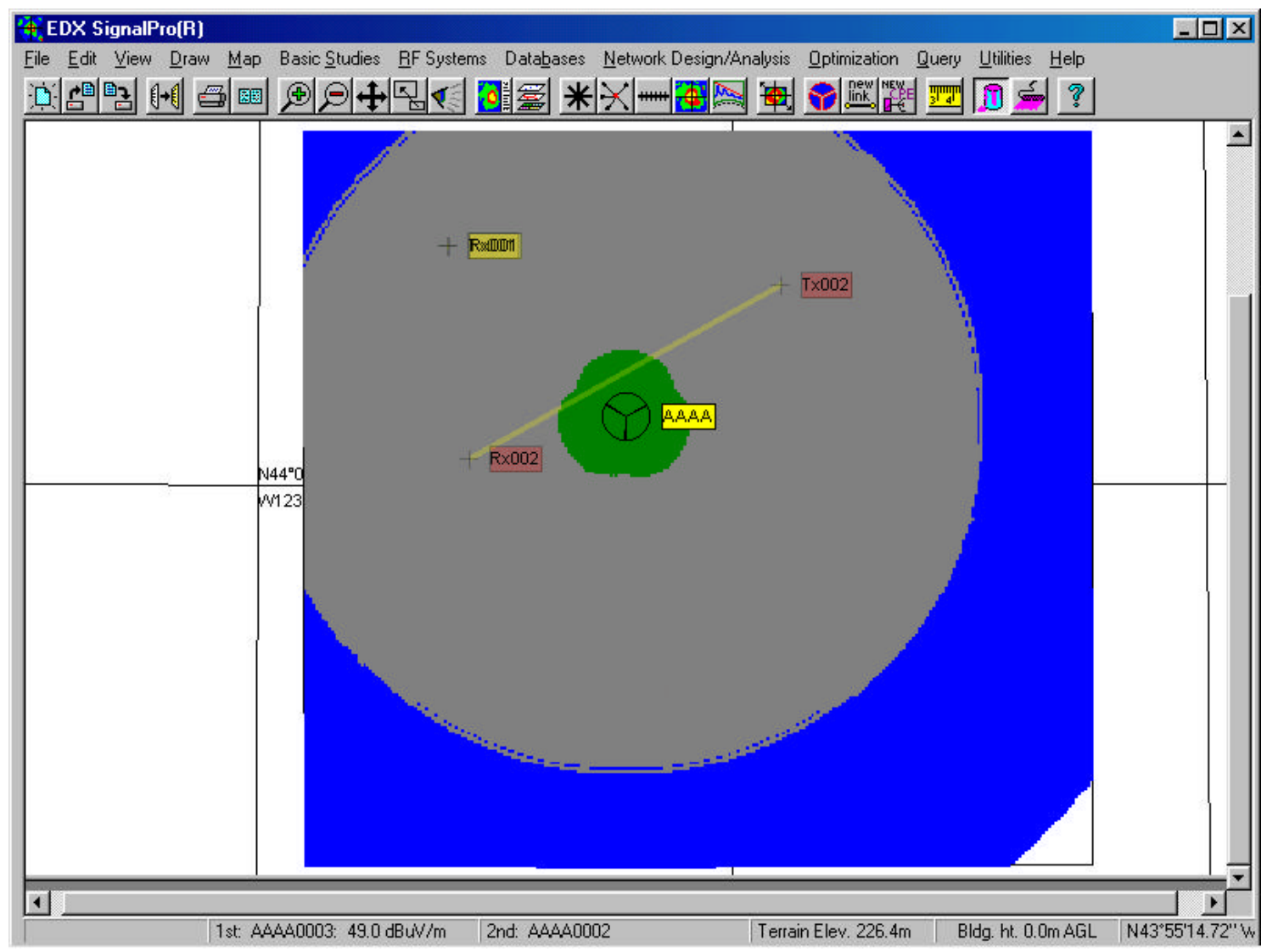

It measures received power at remote for a master group using a free space + RMD propagation model. The difference in the received power is shown by different colors. SignalPro software allows the user to perform link study for link systems to communicate between two distinct points. In cellular communications, building a reliable communication system requires the designer to be able to predict how each system component will perform under varying weather conditions, and in the presence of many types of natural and man-made features which can potentially obstruct the link. It also allows the user to display a profile of the signal link traveling over terrain between the points of a transmitter and receiver. One can then analyze the performance of the link with fade margin and percent availability calculations that take into account all these factors. This is shown in the following link diagram. This study can be performed for multi-link system by either a long distance multi-hop system or a system in a metropolitan area with several links co-existing in the same frequency bands. The software also allows the ability to study the interference among the links. 


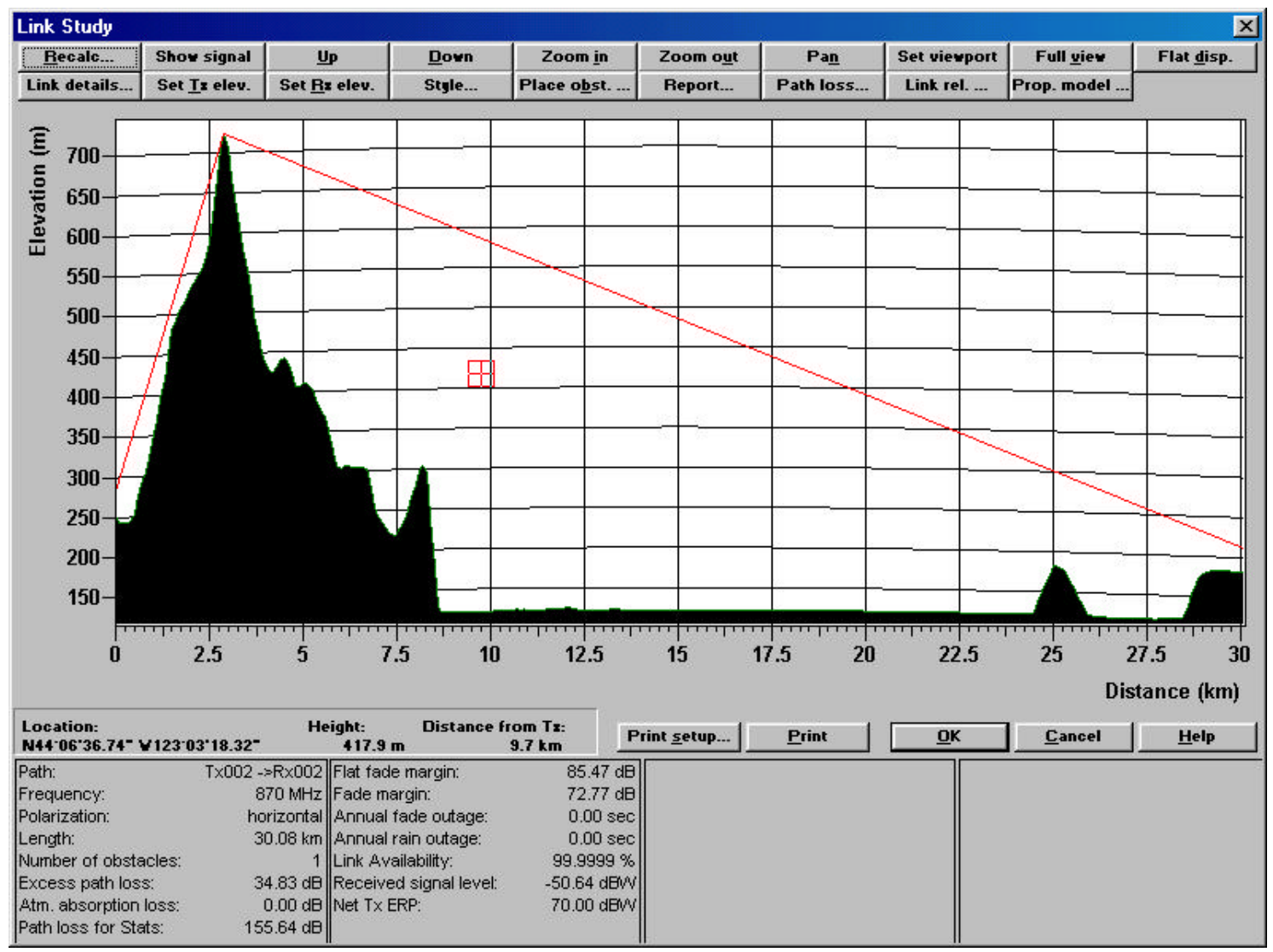

3. System View of ELANIX Inc., was used to study various communication and digital signal processing systems used in the electrical engineering technology courses. The software uses symbolic tokens (functional models) to represent processes, and a time base to represent system sampling characteristics. Simulation of a complete system is designed by selecting tokens from various libraries, connecting them together and defining parameters of the tokens through a dialog box or direct entry of values in graphical representation of circuit schematics. Example of a simple system for the starter included simulation of a sine wave, addition of some noise and then filtering it to obtain a clean sine wave. It also included, obtaining a power spectrum of the simulated signal with and without noise. The block diagram of this example is shown below and is explained step by step in the System View web site (http://www.elanix.com).

Advanced experiments included simulation and study of spread spectrum modulations and 802.11 based wireless local area network. The full version of System View software was donated by Elanix Inc., for classroom usage. A limited version of the software was also provided to the students who could work on their PC at home and could use the full version at the college PC lab.

Proceeding of the 2002 American Society for Engineering Education Annual Conference \& Exposition Copyright (C) 2002, American Society for Engineering Education 
Sorwa: Subond

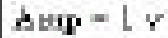

Fue $-107+6$ Hz

Fhas = I tes

Chtwe $0-3 w 1$

Ontpent 1 - C-ain

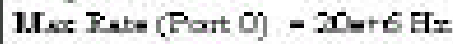

Taken 0!

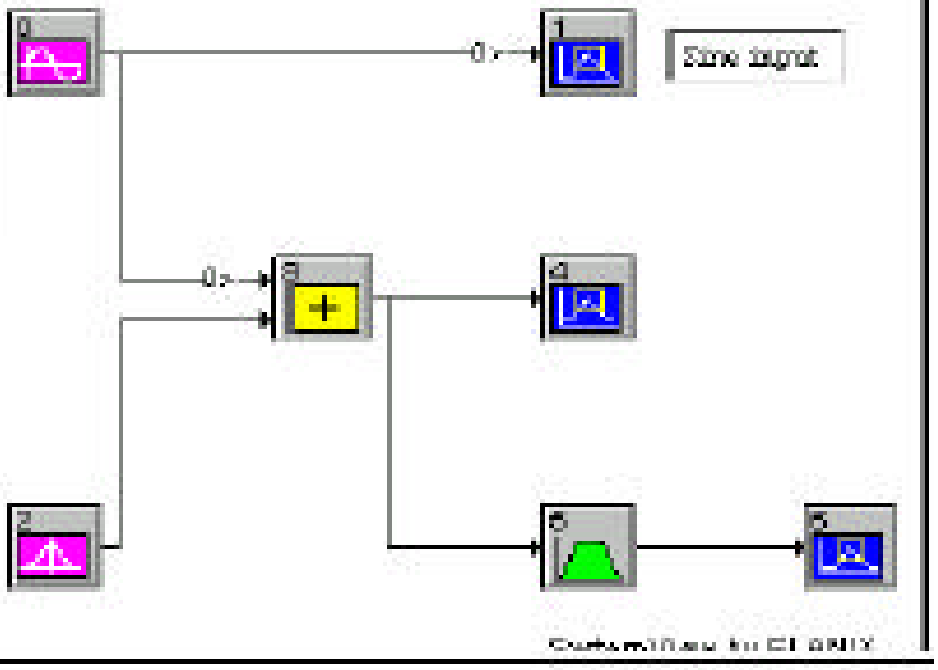

Elanix Inc., web site contains download releases, application notes, examples files and demonstrations. It also lists a schedule of seminars and training courses.

\section{Conclusion}

The use of graphical programming tools to simulate, design, and implement systems for communications, DSPs and control has increased rapidly in industry. Such programming tools are easier to use and are effective teaching tools in learning difficult concepts in electrical engineering and engineering technology courses. The introduction of graphical programming tools in our courses has given the students hands-on experience with the same design tools used by practicing engineers, enhancing their career options in the current technological environment. The professional copies of some of these software packages are expensive, but some of the manufacturers often have special discounted prices for academia and are willing to donate them for teaching purposes.

\section{Acknowledgements}

The authors would like to thank AGX Inc., EDX Inc., and Elanix Inc., for donating the software used in this work. The support of the Information Directorate of the Air Force Research Lab, Rome site, New York, and SUNY Institute of Technology, Utica, New York, is also appreciated.

Proceeding of the 2002 American Society for Engineering Education Annual Conference \& Exposition Copyright (C) 2002, American Society for Engineering Education 


\section{Bibliography}

1. Salahuddin Qazi, "Computer Aided-Design (CAD) and Implementation of DSP

Based Algorithms For Software Radio," Final Report for Summer Faculty Research

Program, Air Force Research Laboratory, Rome Site, New York, September 2000.

2. Salahuddin Qazi, Naseem Ishaq, "Information Technology Education For

Developing Countries and Beginners Using Web-Based Distance Learning,"

Proceeding of 2001 Annual Conference \& Exposition American Society of

Engineering Education, June 2001.

3. http://www.ni.com

4. http://www.mathcom.com

5. http://www.elanix.com

6. http://www.edx.com

7. http://www.hyperception.com

8. http://www.opnet.com

9. http://www.stk.com

10.http://www.rsoft.com

\section{Biography}

SALAHUDDIN QAZI holds a Ph.D. degree in electrical engineering from the University of Technology, Loughborough, England, U.K. and is an Associate Professor in the School of Information Systems and Engineering Technology at the State University of New York Institute of Technology, Utica, New York. He teaches and conducts research in the area of fiber optics, optical communications and wireless communications. He has helped develop and coordinate an undergraduate program in Photonics and a graduate program in Advanced Technology. He is a senior member of IEEE. and a member of American Society of Engineering Education.

NASEEM ISHAQ holds a Ph.D. degree from the University of London, U.K. and is an Associate Professor in the department of electrical engineering technology at the State University of New York Institute of Technology, Utica, New York. Dr. Ishaq's expertise is in the area of data communication \& networking technology, computer \& robotic vision and VLSI design.

Proceeding of the 2002 American Society for Engineering Education Annual Conference \& Exposition Copyright $@$ (2002, American Society for Engineering Education 


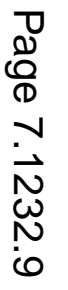

